\title{
Studying Applicability Feasibility of OFDM in Upcoming 5G Network
}

\author{
Nagapushpa K.P \\ Research Scholar \\ Visvesvaraya Technological University \\ Belagavi, Karnataka, India
}

\author{
Chitra Kiran N \\ Prof. \& HOD: Dept. of Electronics \& Communication Sai \\ Vidya Institute of Technology \\ Bangalore, Karnataka, India
}

\begin{abstract}
Orthogonal frequency-division multiplexing (OFDM) is one of unbeatable multiplexing technique till date. However with increasing version of next generation mobile standards like 5G, the applicability of OFDM is quite questionable. The prime reason behind this is in order to offer higher data rates and extensive networking services in $5 \mathrm{G}$, OFDM will be required to overcome some inherent problems of spectral leakage, power consumption, less supportability of increased channel capacity. The reason community still believes that there is positive scope of OFDM to be applicable in 5G network provided it undergoes certain changes. This paper reviews some of the complimentary waveforms that have been theoretically proven to be adding edge to OFDM system. The paper reviews different waveforms as well as multiple access techniques to be used in designing $5 \mathrm{G}$ technology and assess their effectiveness in viewpoint of research applicability and effectiveness towards leveraging $5 \mathrm{G}$.
\end{abstract}

Keywords-OFDM; 5G; Next Generation; Peak to Average Power Ratio (PAPR); multi-carrier; Waveforms; Multiple Access

\section{INTRODUCTION}

5G is basically a standard fifth generation of wireless network designed on the basis of standard IEEE 802.11ac. At present, various enterprises believe that connectivity established on $5 \mathrm{G}$ network is done solely on the basis of performance of a system, quality-of-experience, business models, and enhanced services. Some of the much hyped charecteristics of $5 \mathrm{G}$ technologies are i) availability of thousand times of channel capacity per unit area, ii) offers connectivity of 1-10 Gbps, iii) believed to offer network coverage of $100 \%$, iv) believed to offer 10 years of battery lifetime for low-powered embedded devices, v) can connect around 10-100 time of the devices, vi) offers extremely lowered delay, and vii) offers approximately $99 \%$ of availability. The complete working principle of $5 \mathrm{G}$ technology is based on carrier aggregation, Device-2-Device (D2D) Communication, network access using cloud-radio, usage of smaller cells, Wi-Fi Offloading, Multiple Input Multiple Output (MIMO) concepts. Originally, the concept of carrier aggregation was started in Long Term Evolution (LTE) networks that allow multiple carrier signal to be combined together in order to accommodate the larger channel capacity till $100 \mathrm{MHz}$. The 5G network applies carrier aggregation in the form of three unique approaches called as i) intra-band contiguous, ii) intra-band non-contiguous, and iii) inter-band. The first approach transmits two carriers at the neighboring channels whereas the second approach incorporates channel spacing between two carriers.

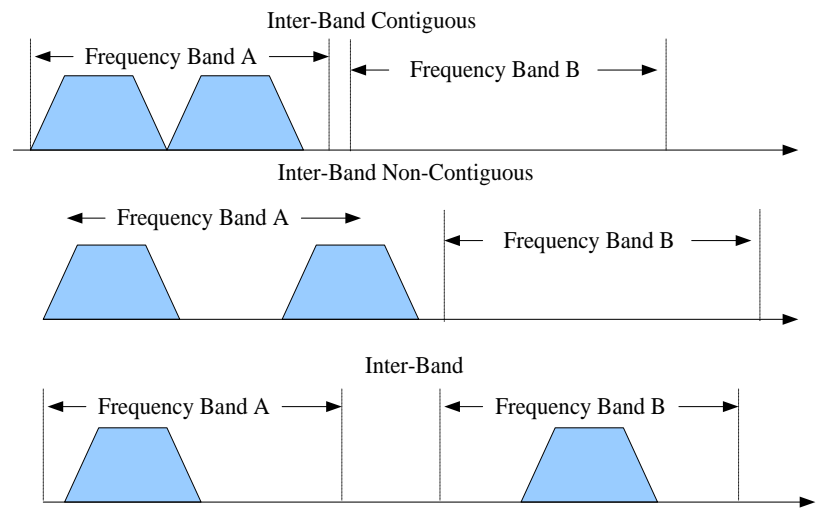

Fig. 1. Concept of Carrier Aggregation in 5G

The third approach uses multiple $4 \mathrm{G}$ bands for parallel transmission. 5G also must support Device-to-Device Communication. This technique allows two different devices residing within communication range of each other to communicate with each other directly. This virtually means that in case the network operator is not working than both the devices can interact with each other seamlessly.

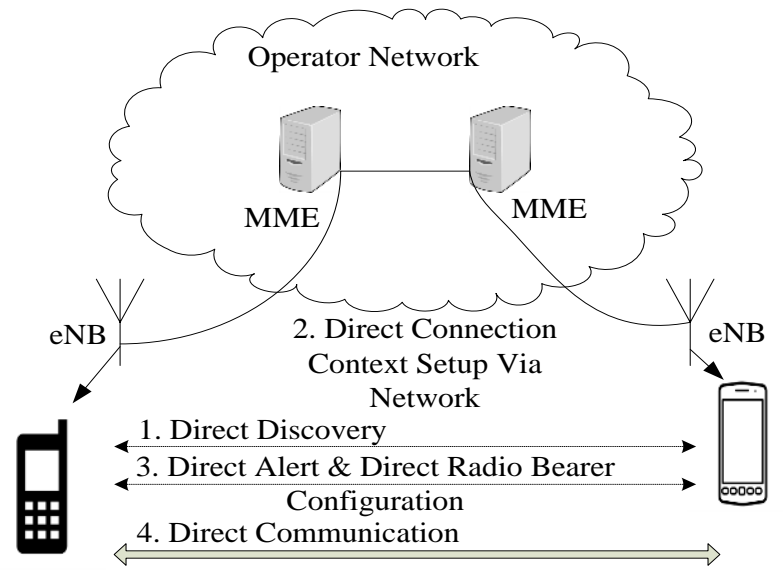

Fig. 2. Concept of D2D Communication

Another most important characteristic of $5 \mathrm{G}$ network is the adoption of accessing network using cloud as well as radio technologies together. 


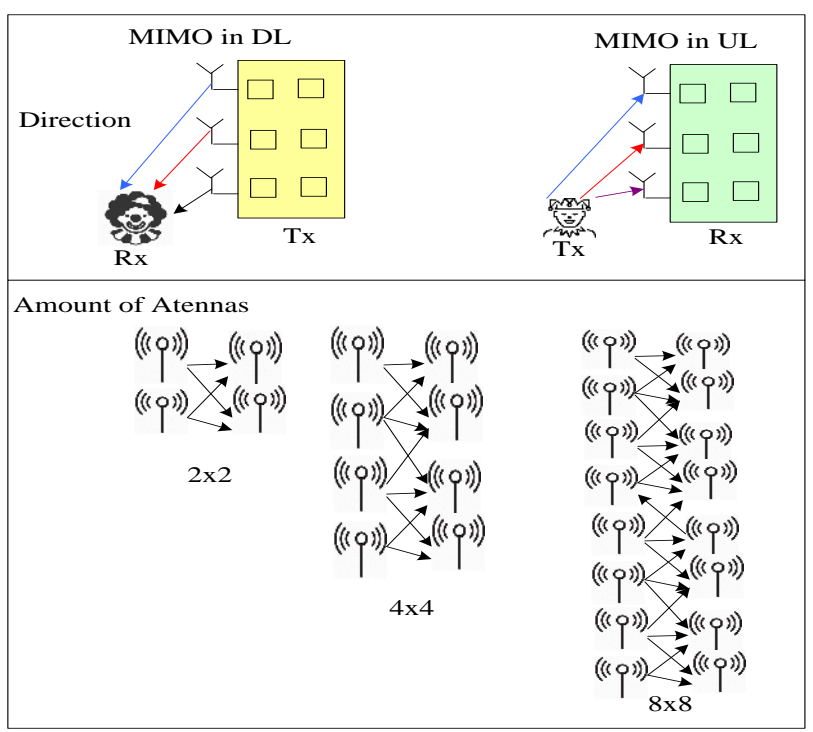

Fig. 3. Concept of MIMO in $5 \mathrm{G}$

This integration mechanism completely supports data processing using centralized approaches that is done from a distance with certain cloud system. Different types of fiber optics are used for establishing a connection among the base station. 5G networks also make use of pico and micro cells for enhancing the network efficiency. It also supports reusability of spectrum by integrating increasing number of users for effectively managing the networks. Usage of Wi-Fi network and integrating with the mobile network is another working principle of 5G networks and thereby it assists in establishing communication where quality of network is poor. Finally, MIMO (Multiple Input Multiple Output) are also used in 5G network in order to increase its data rates. This technology allows transmission of parallel data transfers and thereby data rates are increased even compared to present LTE networks and other conventional next generation networks e.g. $2 \mathrm{G}$ and $3 \mathrm{G}$.

OFDM is obviously a most powerful multiplexing technique that has some potential advantages till $3 \mathrm{G}$ networks but limit itself from LTE networks onwards. Hence, it is imperative to investigate the potential complimentary waveforms generated by amending conventional OFDM. Therefore, this paper reviews about different techniques of how OFDM can be enhanced to make itself suitable to hold $5 \mathrm{G}$ services with respect to existing research approaches. Table.1 highlights the comparative differences in the different next generation standards. Section II discusses about the diverse trends of $5 \mathrm{G}$ waveforms followed by trends of $5 \mathrm{G}$ access schemes in Section III. Section IV discusses about the study findings and research gap discussion followed by conclusion in Section V.

TABLE I. COMPARISON OF DIFFERENT GENERATIONS OF MOBILE

\begin{tabular}{|c|c|c|c|c|c|c|}
\hline Standards & Access technology & Application & Bandwidth & $\begin{array}{l}\text { Frequency } \\
\text { Band }\end{array}$ & Data rate & FEC \\
\hline $1 \mathrm{G}$ & FDMA, AMPS & Voice & $30 \mathrm{KHz}$ & $800 \mathrm{MHz}$ & $2.4 \mathrm{kbps}$ & N/A \\
\hline \multirow{4}{*}{$2 \mathrm{G}$} & CDMA & \multirow{4}{*}{ Voice, Data } & $1.25 \mathrm{MHz}$ & \multirow{4}{*}{$\begin{array}{l}850-900-1800- \\
1900 \mathrm{MHz}\end{array}$} & $10 \mathrm{kbps}$ & \multirow{4}{*}{ N/A } \\
\hline & TDMA, GSM & & $200 \mathrm{KHz}$ & & $10 \mathrm{kbps}$ & \\
\hline & GPRS & & $200 \mathrm{KHz}$ & & $50 \mathrm{kbps}$ & \\
\hline & EDGE & & $200 \mathrm{KHz}$ & & $200 \mathrm{kbps}$ & \\
\hline \multirow{6}{*}{$3 \mathrm{G}$} & UMTS, WCDMA & \multirow{4}{*}{$\begin{array}{l}\text { Voice, video } \\
\text { calls, data service }\end{array}$} & $5 \mathrm{MHz}$ & \multirow{4}{*}{$\begin{array}{l}850-900-1800- \\
1900-2100 \mathrm{MHz}\end{array}$} & $384 \mathrm{kbps}$ & \multirow{4}{*}{ Turbo Codes } \\
\hline & CDMA2000 & & $1.25 \mathrm{MHz}$ & & $384 \mathrm{kbps}$ & \\
\hline & HSDPA, HSUPA & & $5 \mathrm{MHz}$ & & $30 \mathrm{Mpbs}$ & \\
\hline & EVDO & & $1.25 \mathrm{MHz}$ & & $30 \mathrm{Mpbs}$ & \\
\hline & $\begin{array}{l}\text { OFDMA, SC-FDMA, } \\
\text { LTE }\end{array}$ & \multirow{2}{*}{$\begin{array}{l}\text { HD TV, online } \\
\text { gaming }\end{array}$} & $20 \mathrm{MHz}$ & $1.8-2.6 \mathrm{GHz}$ & $200 \mathrm{Mbps}$ & \multirow{2}{*}{$\begin{array}{l}\text { Concatenated } \\
\text { Codes }\end{array}$} \\
\hline & SOFDMA, WiMaX & & $7-10 \mathrm{MHz}$ & $3.5-5.8 \mathrm{GHz}$ & $200 \mathrm{Mbps}$ & \\
\hline \multirow{2}{*}{$4 \mathrm{G}$} & $\begin{array}{l}\text { LTE-A, OFDMA/SC- } \\
\text { OFDMA }\end{array}$ & \multirow{2}{*}{$\begin{array}{l}\text { HD TV, online } \\
\text { gaming }\end{array}$} & $20 \mathrm{MHz}$ & $1.8-2.6 \mathrm{MHz}$ & $\begin{array}{l}3 \text { Gbps(DL) } \\
1.5 \text { Gbps (UL) }\end{array}$ & \multirow{2}{*}{ Turbo Codes } \\
\hline & SOFDMA, WiMaX & & $3.5-8.75 \mathrm{MHz}$ & $2.3-2.5 \mathrm{GHz}$ & $200 \mathrm{Mbps}$ & \\
\hline $5 \mathrm{G}$ & BDMA, FBMC & $\begin{array}{l}\text { Virtual reality, } \\
\text { UHD video }\end{array}$ & $60 \mathrm{GHz}$ & $1.8-300 \mathrm{GHz}$ & $50 \mathrm{Gbps}$ & LDPC \\
\hline
\end{tabular}

II. TRENDS OF 5G WAVEFORMS

Basically, the derivation of the $5 \mathrm{G}$ services is said to be originated from technology named Enhanced Mobile Broadband (eMBB) [1]. This is because eMBB offers minimal latency and is proven highly scalable. It can be also used for structuring sub-frame that is self-contained for both unlicensed as well as licensed spectrum. It also offers an integrated access to device-to-device communication. Usage of eMBB in $5 \mathrm{G}$ technology will be seen in millimeter wave, wide area IoT, and networking services with higher reliability. eMBB can be used for millimeter wave to offer common media access control (MAC) usage, beam tracking of millimeter wave, backhaul and access while eMBB can be used in IoT for offering waveforms with minimal energy, reduced overheads, link with higher optimization, and managed mesh. Similarly, eMBB can be used to develop a network service. Hence, it is believed that usage of eMBB in 5G design principle offers some significant advantage e.g. i) enhanced spectral efficiency, ii) maximized throughput, iii) offers flexible optimization schemes for particular deployment scenario, and finally iv) it is known to reduce the control as well as signaling overhead in order to enhance the efficiency. Figure.4 outlines the role and contribution of eMBB on three different scenarios of deployment in 5G technology. Apart from the above mentioned benefits of eMBB, the prime target of $5 \mathrm{G}$ waveforms are to offer minimal consumption of power, reduced in-band as well as out-of-band emission, supporting multiple access with asynchronous types, and finally maximized spectral efficiency. 


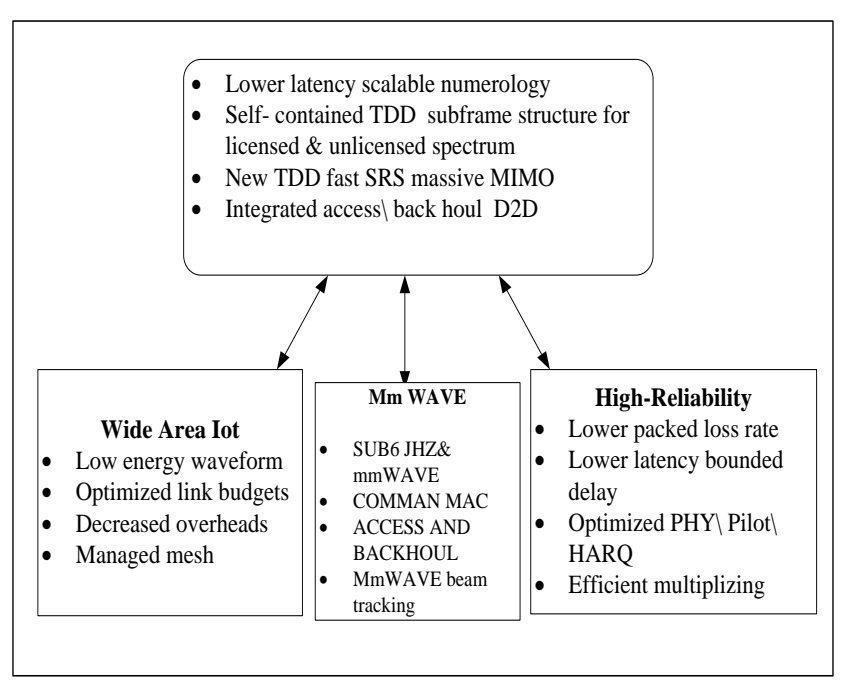

Fig. 4. Role of eMBB in 5G Technology

The 5G waveforms should support MIMO as well as it must also deal with interference problems. The scalability is believed to be improved by reducing the protocol overhead, maximized capacity, and minimized consumption of power. This section will now briefly elaborate about the different types of waveforms in $5 \mathrm{G}$ technology.

1) Orthogonal Frequency Division Multiplexing (OFDM):

Basically, OFDM is a modulation method of digital multiple carriers [2] that is characterized by its ability to sustain adverse channel condition in wireless networks. It is also known to offer maximized spectral efficiency with implementation of fast Fourier Transforms (FFT). The basic operation of OFDM is shown in Figure 5. Different from convention frequency-division multiplexing (FDM) schemes, it doesn't require tuned receivers for sub-channels. Examples of the waveform supported by OFDM-based multi-carriers are CP-OFDM (this is adopted in LTE specification), CP-OFDM with / without WOLA (this is existing implementation of LTE), Universal Filtered Multi-Carrier (UFMC), Filter Bank Multicarrier (FBMC), and Generalized Frequency Division Multiplexing (GFDM).

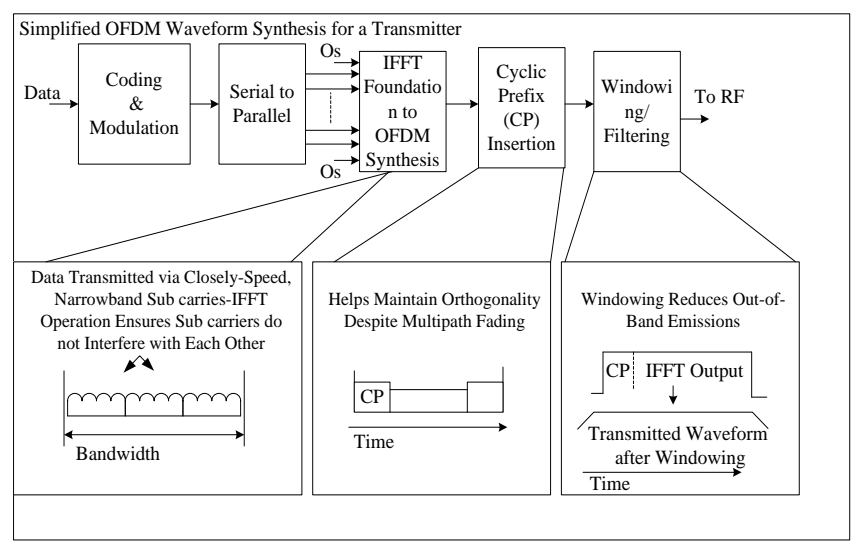

Fig. 5. Operations of OFDM
The existing research work done towards OFDM scheme and its associated limitations are as highlighted:

- Existing Research Contribution: Studies towards usage of OFDM in 5G is quite less. In last five years (20112016), there are only 66 journals in IEEE which directly discusses applicability of OFDM in 5G technology. OFDM was used for applying light fidelity technology in 5G by Abdallah and Boudriga [3]. Venkatesan and Valenzuela [4] have introduced a study that uses cyclic prefix as well as zero prefix as the optional method as guard interval. The study has also enhances the decay rate of spectrum using both windowing as well as filtering scheme over the receiver for resisting interference. Wang et al. [5] have addressed the problem of non-linear impairment of OFDM in 5G networks. The authors have used millimeter wave for investigating the level of interference existing on OFDM using both theoretical and experimental analysis. Wang et al. [6] have presented a self-contained transmission for offering enhanced channel capacity in $5 \mathrm{G}$ networks. The technique uses pulse shaping over time domain. Bogucka et al. [7] have introduced a mechanism of aggregating spectrums of dynamic nature in 5G. Loulou and Renfors [8] have presented a study where a comparative analysis of existing suppression techniques and to overcome the spectral sidelobes of high power in OFDM. Lin et al. [9] have used MachZehnder modulator for processing incoming signal into double sideband using experimental approach. Li et al. [10] have discussed a study where a hardware-based platform has been designed to achieve symbol synchronization. A new scheme called as FC (Flexible Configured)-OFDM is presented by Lin et al. [11] for configuring sub-bands in 5G usage. Kildal et al. [12] have used both MIMO as well as OFDM for increasing throughput in 5G networks. However, the study is more focused on investigating its effect on Line of Sight performance for 5G antenna. Banelli et al. [13] have presented a discussion of different modulation schemes which proved that although OFDM is not self-capable to meet complete demands of $5 \mathrm{G}$, but when other forms of waveforms comes as an compliments to OFDM, its potential increases multi-folds.

- Limitation of Existing Schemes: However, OFDM is highly sensitive to issues of frequency synchronization as well as Doppler shift. It is also known for its maximized PAPR (Peak-to-Average Power Ratio) followed by minimized efficiency due to guard interval (or cyclic prefix).

2) Single Carrier quadrature amplitude modulation QAM $(S C-Q A M)$

This kind of waveform possesses an advantage of minimal PAPR and reduced spectral leakage. It also supports asynchronous multiplexing. Figure.6 showcase the transmitter and receiver design used for single carrier QAM (i.e. SCQAM) 


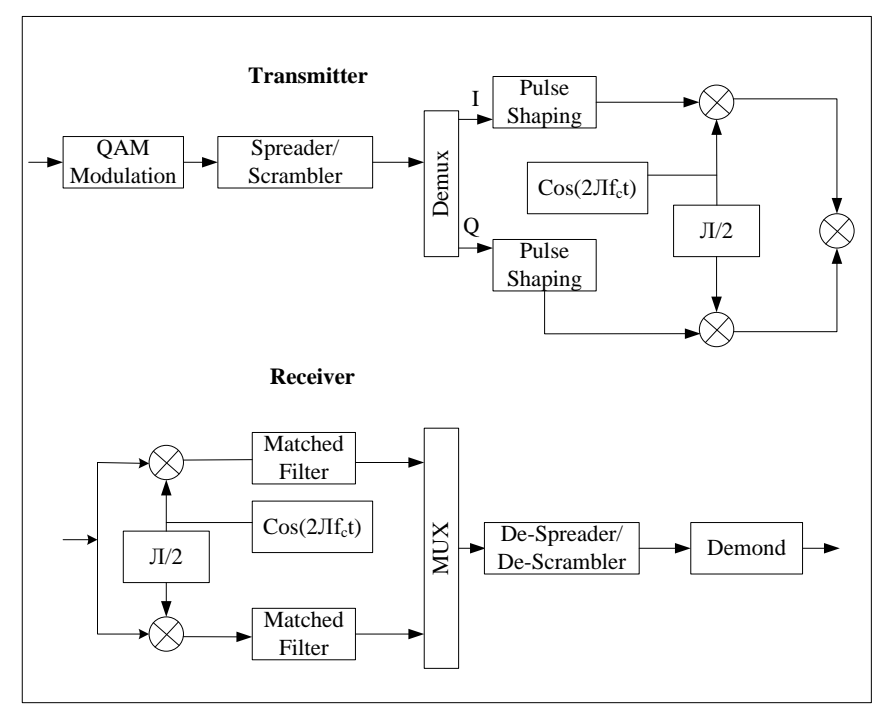

Fig. 6. Operations of Single Carrier QAM

The existing research work done towards SC-QAM scheme and its associated limitations are as highlighted:

- Existing Research Contribution: There are very much limited research work being carried out where SCQAM was found to be used for $5 \mathrm{G}$ technologies. A total of 89 journals were found in IEEE where SC-QAM was used, but very few papers exists to say that it was used exclusively for $5 \mathrm{G}$ technologies. Majority of the techniques has used hardware-based approach. Adoption of SC-QAM was found in study of Deng et al. [14], Duyen [15], and Zhang et al. [16]. SC-QAM was used for software defined MIMO, assessing performance of MIMO, enhancing performance of optical network. Although, all these research works presents a good guidelines, but its potential towards $5 \mathrm{G}$ technology is yet to be investigated in true sense.

- Limitation of Existing Schemes: Such schemes are found not to extensively support MIMO concepts. It is also associated with quite restricted flexibility while allocating spectrums. Another bigger problem is its increased complexity of the receiver owing to usage of equalization approaches for enhancing spectral efficiency. $F D E$ )

3) Single Carrier Frequency Domain Equalization (SC-

This scheme is exactly same as that of SC-QAM with a difference that it adds Cyclic Prefix (CP) as shown in Figure.7. The prime motivation behind using or adopting such scheme is its applicability of frequency domain equalization even in single carried waveforms. The scheme was also found to enhance spectral efficiency even when the waveform is subjected to multipath fading.

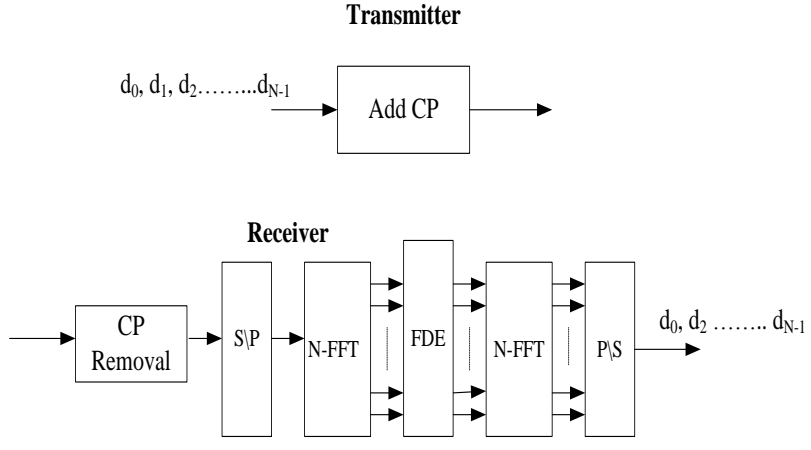

Fig. 7. Operations of Single Carrier QAM

The existing research work done towards SC-FDE scheme and its associated limitations are as highlighted:

- Existing Research Contribution: There are total of 55 journals published in last 5 years in IEEE that has used SC-FDE and there are only 2 conferences that actually have attempted to associate SC-FDE with 5G technology. Ribeiro et al. [17] have used SC-FDE for uplink waveform generation while downlink was generated by OFDM. The base idea is to enhance the base station cooperation system in $5 \mathrm{G}$ networks. SCFDE was also used for modulation in MIMO-based schemes as seen from the investigation presented by Dinis and Montezuma [18] for designing iterative receiver. It was also found to be used for selecting the position of pilot as well as reconstructing signal in the work carried out by Zheng et al. [19]. The technique was used for minimizing inter-symbol interference as well as to check for lowered computational complexity. SC-FDE was also used for compensating the pass-loss in millimeter wave by integrating with OFDM. This work was carried by $\mathrm{Wu}$ et al. [20] to offer lower PAPR. Similar work direction was carried out by Cheng et al. [21] towards investigating imbalance between inphase and quadrature. SC-FDE was also used for minimizing PAPR over time domain by Boonkajay and Adachi [22] using principle of selective mapping.

- Limitation of Existing Schemes: The significant pitfall of this scheme is that adding cyclic prefix leads to declination of the spectral efficiency to certain extent. This scheme also suffers from spectral leakage problems (it is found to be even higher than SC-QAM scheme).

\section{4) Single Carrier FDM (SC-FDM)}

Single Carrier Frequency Division Multiplexing is known for its supportability in assignment of channel capacity in dynamic manner. It uses frequency multiplexing in order to offer flexibility in allocation task for multiple users. It is also known for its capability to countermeasure degradation due to multipath propagation. 


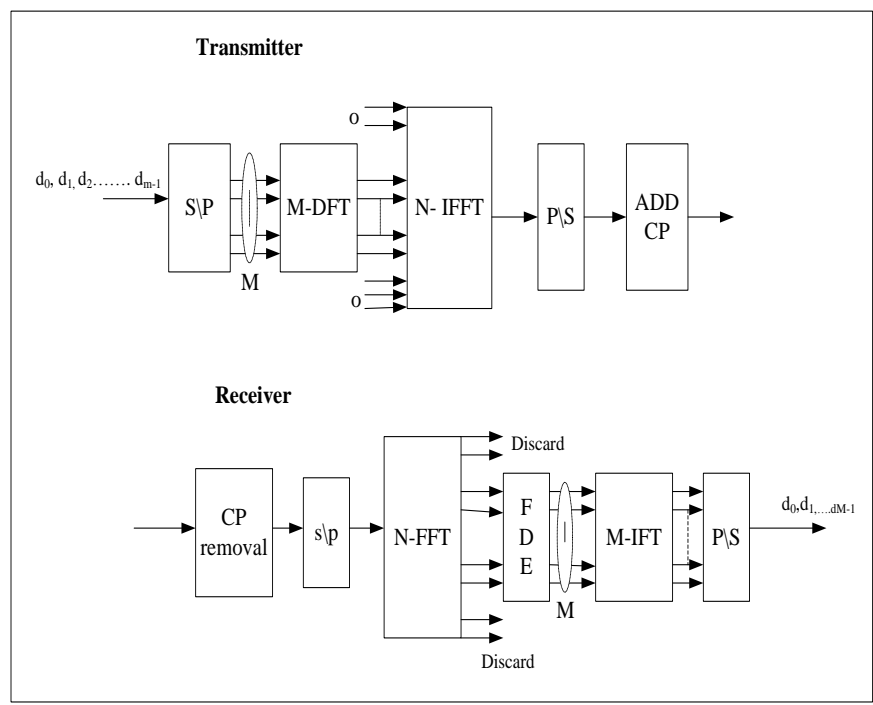

Fig. 8. Operations of Single Carrier FDM

The existing research work done towards SC-FDM scheme and its associated limitations are as highlighted:

- Existing Research Contribution: Existing research work towards using SC-FDM is extremely less. There are only 2 journals and 9 conferences in IEEE pertaining to use of SC-FDE published in last 5 years. SC-FDM was used in minimizing PAPR and thereby performs optimization. This is seen in the work carried out by Zhao et al. [23]. Similar direction of research work was also carried out by Luo et al. [24]. The technique allows multiplexing pilots and data within SC-FDM without disturbing the waveform of single carrier. It was also found to estimate coherent channel in LTE networks. SC-FDM was also used in optical network by Zhou et al. [25] for low-complexity multiplexing as well as reduction of PAPR too. Kobayashi et al. [26] have used SC-FDM for enhancing the tolerance level on optical network. The technique was found to compensate nonlinear effects in conventional multi-carrier channels.

- Limitation of Existing Schemes: The potential limiting factor of such scheme is maximized PAPR as well as higher spectral leakage even as compared to SC-QAM scheme. It has also higher dependability of synchronous multiplexing operation.

5) Weighted Overlap and Add (WOLA)

In this method, an additional window (also known as synthesis window) is applied after performing an inverse DFT operation and before obtaining final overlap-add in order to accomplish output signal. Such forms of output windows play a crucial role in reducing blocking effect. All sorts of spectral coding error are minimized by synthesis window at the frame boundary. Usage of WOLA is more frequent in non-linear FFT processor with instantaneous operation. Therefore, WOLA is preferred in the cases when there is a need of suppressing interference in out-of-bands and in-band waveforms. One of the significant advantages of using WOLA with other scheme is its capability to control spectral leakage over time-domain windowing. The scenario of implementation of WOLA in time-domain can be seen in Figure.9.

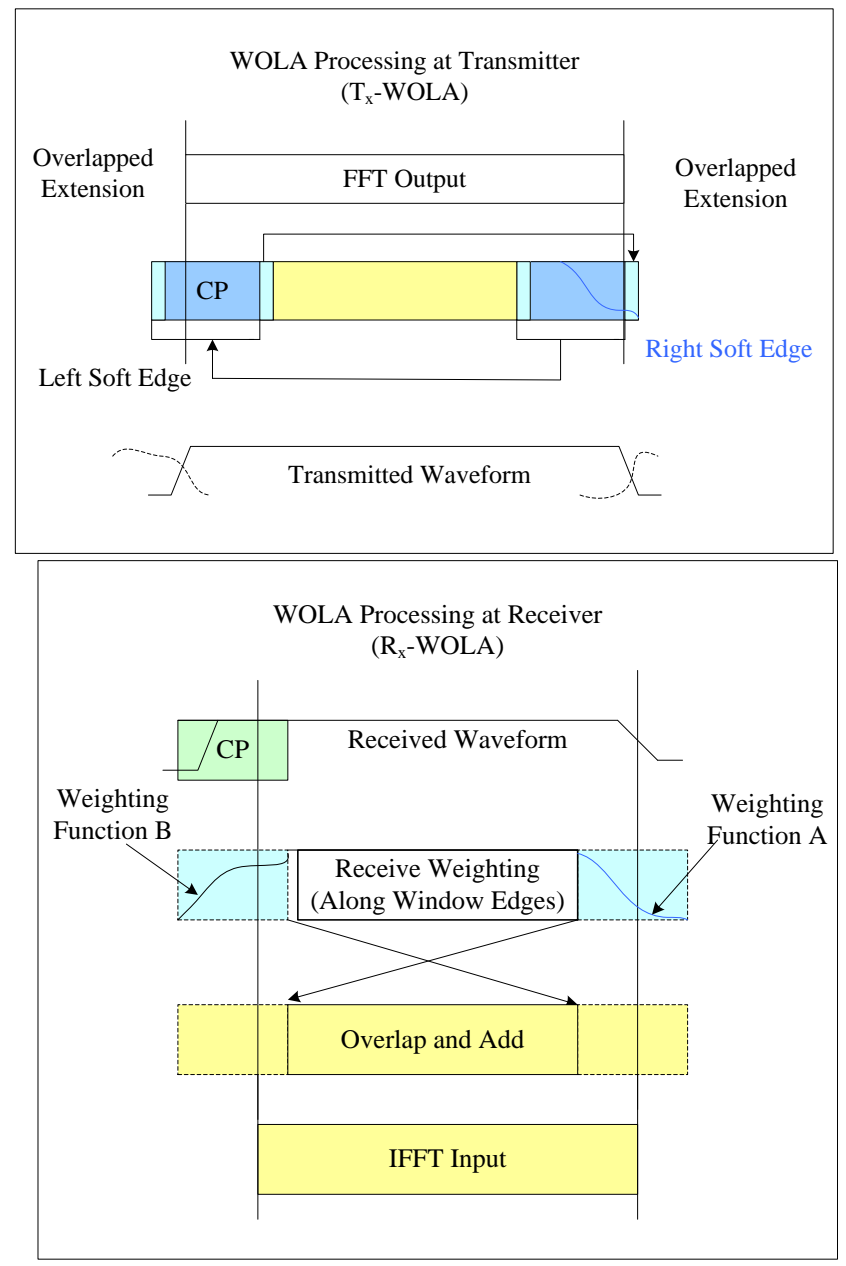

Fig. 9. Operations of WOLA

The existing research work done towards WOLA scheme and its associated limitations are as highlighted:

- Existing Research Contribution: Research work pertaining to WOLA is also very less to find. There are only 5 conference papers in IEEE published in last 5 years. WOLA was used for enhancing performance of multi-channel signals by Doorknob et al. [27]. The authors have designed a filter for studying the frequencies. Usage of WOLA was also seen in passive optical network witnessed in the work of Frey et al. [28] with focus on coherent access network. Similar direction of the work is also carried out by Klionskiy et al. [29] for monitoring hydroacoustic. WOLA was also used for suppressing noise in microphone applications as seen in the work carried out by Lai et al. [30]. Usage of WOLA was seen in the form of filter for overcoming the problem of DFT structure in work carried out by Tao et al. [31].

- Limitation of Existing Schemes: One of the significant limitations of WOLA is that it is highly dependent on 
channels that are spaced evenly and need uniform filtering.

\section{6) Zero-Tail SC-FDM}

Zero-Tail SC-FDM is another scheme that is known for its wider scope of allocating channel capacity. This scheme is completely free from using cyclic prefix and also supports maximized spectral efficiency. The problem of out-of-band leakage can be significantly suppressed in this scheme. The scheme along with its transmitter and receiver design is shown in Figure.10.

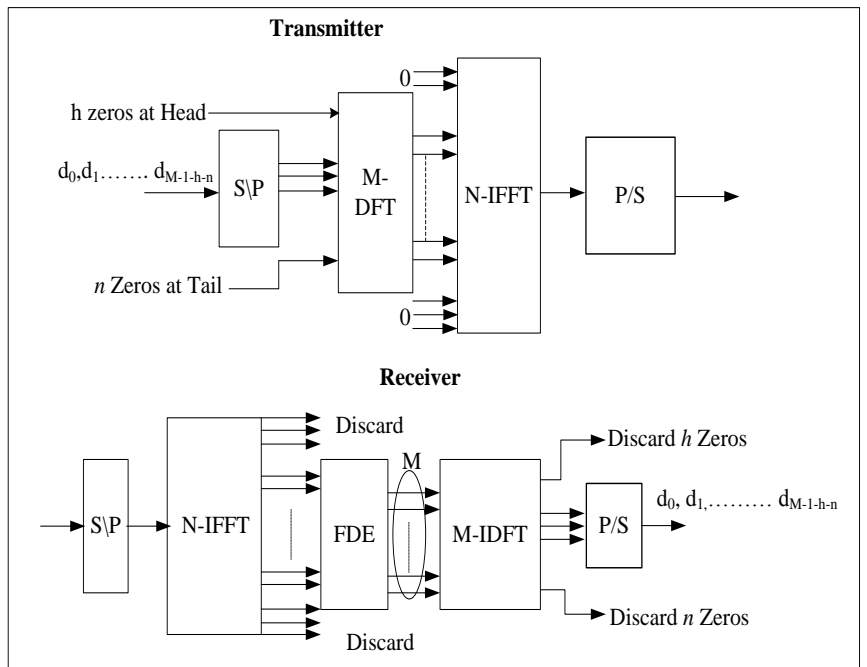

Fig. 10. Operations of Zero-Tail SC-FDM

The existing research work done towards Zero-tail SCFDM scheme and its associated limitations are as highlighted:

- Existing Research Contribution: There is no direct research paper where zero-tail SC-FDM was used for $5 \mathrm{G}$ technology. But there is only one author has deliberately investigated on this topic. Berardinelli et al. [32] have used zero tail DFT in order to substitute cyclic prefix in OFDM. Applicability of similar technique to improve and make OFDM suitable for $5 \mathrm{G}$ network was discussed by same authors in [33] and [34]. The authors have adopted analytical approach in order to mitigate the overhead as well as propagation delay. Pazos [35] have published a report that has discussed about tail generation process, which is merely a theoretical discussion.

- Limitation of Existing Schemes: Although, these scheme is found to possess better suppression performance of out-of band spectrum compared to conventional DFT based OFDM, but this scheme is not found to outperform DFT based OFDM using WOLA scheme. Zero-tail SC-FDM also suffers from additional overhead of signaling in order to set its zero-tail. This scheme also offer lowered flexibility while multiplexing with OFDM due to absence of cyclic prefix as it as all symbol sizes with difference. Moreover, it is highly dependent on synchronous multiplexing.

7) CP-OFDM waveform
This scheme uses cyclic prefix along with supportability of implementing FFT as well as IFFT operations. Flexible allocation of spectrum is another characteristics of this scheme. One of the best parts of this scheme is its direct applicability towards MIMO technology to offer better data rates as well as multiplexing performance. It also offers simple frequency division equalization towards mitigating interference arising from multipath propagation. The working principle of this scheme with respect to transmitter and receiver is as shown in Figure.11

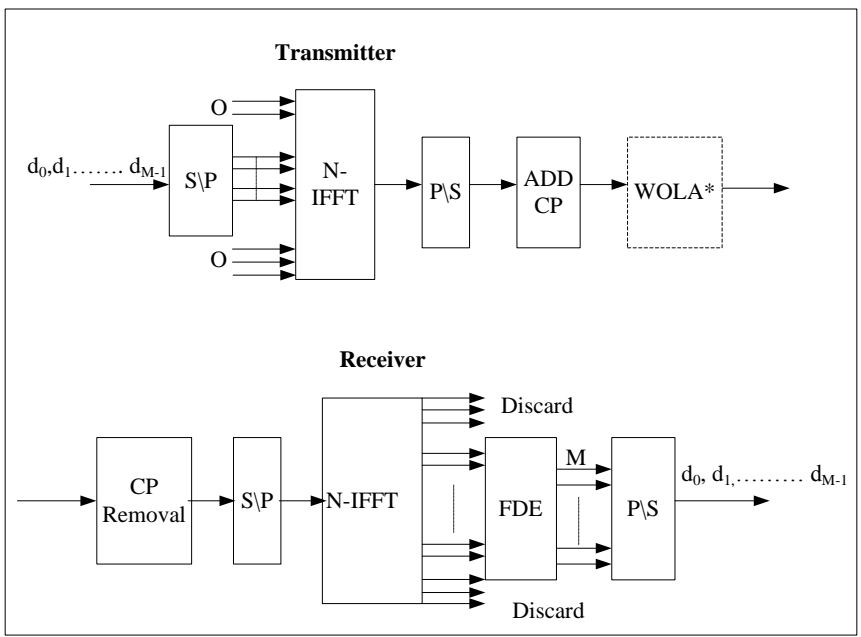

Fig. 11. Operations of CP-OFDM

The existing research work done towards CP-OFDM scheme and its associated limitations are as highlighted:

- Existing Research Contribution: Research work towards CP-OFDM too exists in a smaller scale in the direction of 5G. CP-OFDM was studied by various authors e.g. Ahmed et al. [36], Bodinier et al. [37], Renfors et al. [38], Bellanger et al. [39], Chen et al. [40], Katselis [41], and Waterschoot et al. [42]. The approaches are majorly in the direction of optimizing uplinks, mitigating interference, enhancing convolution, supporting FFT in cognitive radio, addressing synchronization problems in OFDM, addressing PAPR issues, optimizing power spectral density. Although, all these studies have some potential contribution, but there is still a less evidence to claim if CP-OFDM is the best option for designing upcoming $5 \mathrm{G}$ technologies. It has both advantages as well as unsolved issues associated with it.

- Limitation of Existing Schemes: This scheme significantly suffers from inferior localization of frequencies owing to usage of rectangular filter prototype. Although, its performance of controlling leakage can be improved using WOLA but it exhibits poor performance without WOLA.

8) Universal-Filtered Multi-Carrier (UFMC)

This kind of waveform is basically developed by enhancing waveforms generated by CP-OFDM. The scheme performs splitting of the signal in to various sub bands that are then subjected to filters. Usage of cyclic prefix is absent in UFMC 
and it is replaced by using guard interval of zeros that are added in between IFFT symbols. This operation significantly controls inter-symbol interference levels. UFMC is also characterized by equivalent leakage suppression performance just like CP-OFDM when used with WOLA. UFMC can be also applied for multiplexing number of users with heterogeneous numerologies. The working principle of UFMC with transmitter and receiver can be seen in Figure.12.

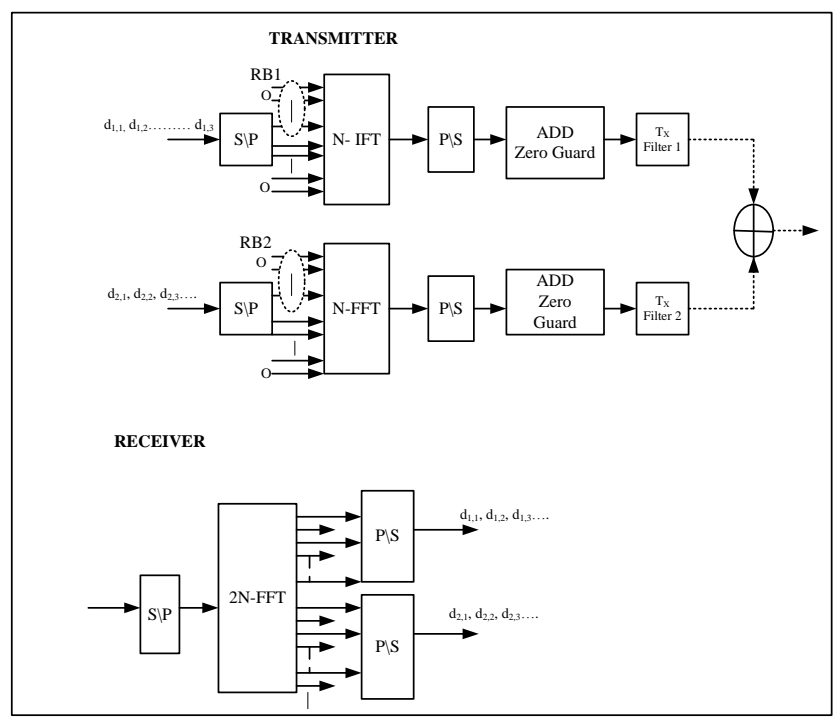

Fig. 12. Operations of UFMC

The existing research work done towards UFMC scheme and its associated limitations are as highlighted:

- Existing Research Contribution: Presence of only 3 journals and 24 conference papers in IEEE for UFMC implementation shows that it is still in infancy stage of implementation. Some of the researchers who have initiated investigation towards UFMC are Kim et al. [43], Vitiello et al. [44], Zhang et al. [45], Geng et al. [46], Mukherjee et al. [47], and Schaich et al. [48] who have addressed the problems of resource management, analysis of performance, optimizing filter performance for out of band emission, etc. There is no direct implementation towards claiming UFMC adhering to $5 \mathrm{G}$ standards in any of the research work.

- Limitation of Existing Schemes: Universal Filtered Multi-Carrier (UFMC) is associated with a loophole of complex design principle of receiver and transmitter. Owing to absence of cyclic prefix, UFMC suffers from inter-symbol interference.

9) Filter Bank Multi-Carrier (FBMC)

This is one of the most preferred selections of waveform especially when it comes to $5 \mathrm{G}$. This scheme performs filtering of only the sub-carriers individually and doesn't filter the complete bands. Better outcomes of spectral efficiency can be ensured by FBMC as it doesn't make use of cyclic prefix. One of the significant advantages of this scheme is its highly improved side lobe decay as compared to any conventional multi-carrier waveforms. The working principle of FBMC with transmitter and receiver can be seen in Figure.13.

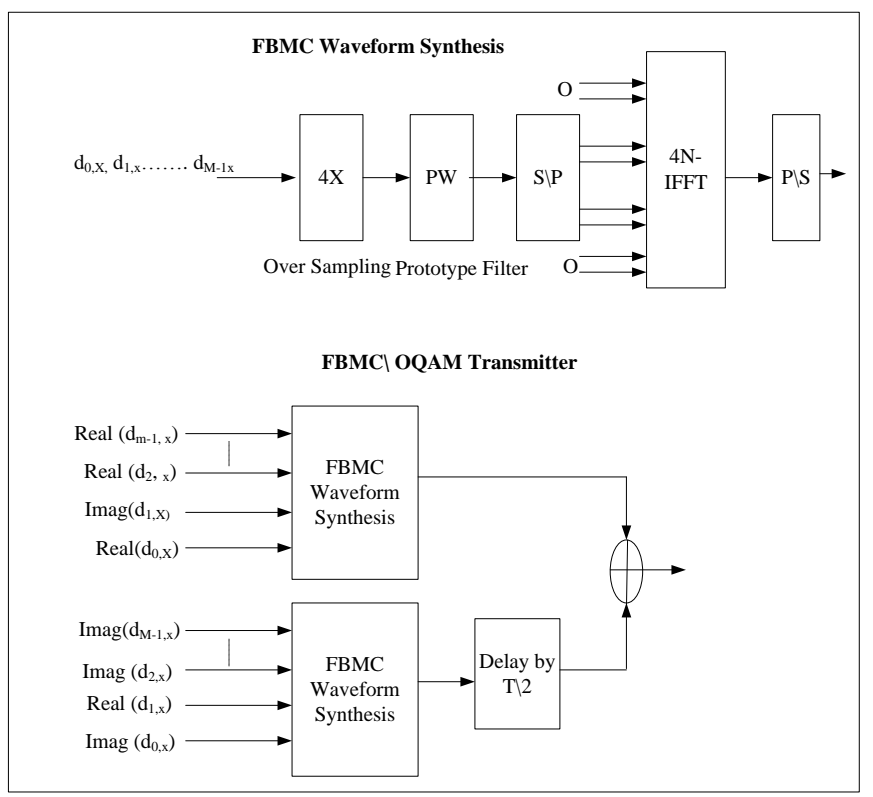

Fig. 13. Operations of FBMC

The existing research work done towards FBMC scheme and its associated limitations are as highlighted:

- Existing Research Contribution: Adoption for FBMC is also few to be found used in $5 \mathrm{G}$ implementation. Adoption of FBMC was seen in the work of Hosseini et al. [49], Jamal [50], Mestre [51], $\mathrm{Na}$ et al. [52], Rottenberg et al. [53], and Sim et al. [54]. All these works have addressed the problems of spectral leakage in millimeter wave, mitigate interference in communication between air and ground, inter-carrier interference, frequency selectivity, etc.

- Limitation of Existing Schemes: This scheme suffers from complex receiver design. It was also found to be not resilient against inter-symbol interference when working on non-flat channels. Moreover, this scheme has highly complicated design principle of using MIMO.

10) Generalized frequency division multiplexing (GFDM)

This scheme is quite similar to legacy OFDM scheme with a difference that its carriers are never orthogonal (like in OFDM, the carriers are always orthogonal). This scheme is known for its capability of controlling various emissions (outof-band) and minimized the PAPR too. GFDM is also similar to FBMC with a difference that a block is maintained for multiple OFDM symbols and a cyclic prefix is added on such blocks. In order to achieve varied OFDM symbols, a prototype filter is subjected to cyclic shift within the block. GFDM scheme is found to be superior to CP-OFDM scheme with respect to leakage suppression. The working principle of GFDM with transmitter and receiver can be seen in Figure.14. 


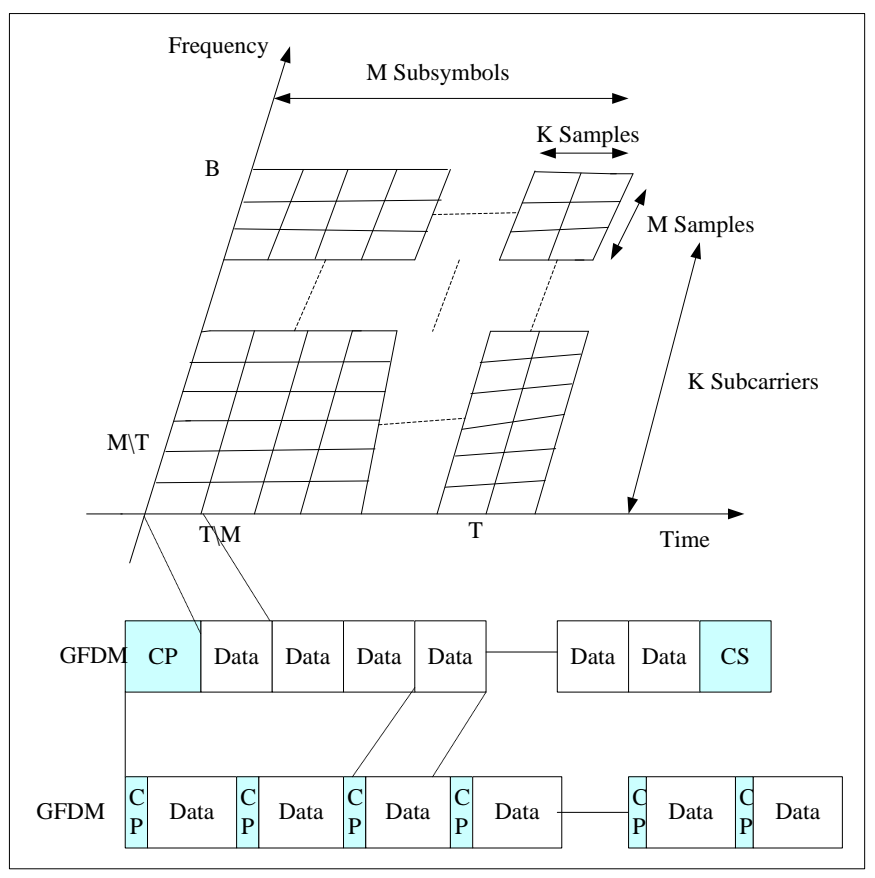

Fig. 14. Operations of GFDM

The existing research work done towards GFDM scheme and its associated limitations are as highlighted:

- Existing Research Contribution: The existing literatures on GFDM has been carried out for addressing problems of dealing with uncertainties in carrier frequencies, PAPR reduction, singularity issue in pulse shaping, synchronization, interference in multicarrier signals, identification of potential MIMO-based signals. Such ground work has been carried out by Chang et al. [55], Sharifian et al. [56], Lin et al. [57], Wang et al. [58], Wei et al. [59], and Zhang et al. [60].

- Limitation of Existing Schemes: It bears the similar problem as that of FBMC i.e. complicated receiver design. Usage of prototype filter further complicates the modulation scheme. GFDM is also witnessed to maximized latency during block processing. It also requires a higher guard band in order to perform multiplexing operation with CP-OFDM.

\section{TRENDS OF 5G ACCESS SCHEMES}

There are varied forms of the $5 \mathrm{G}$ access schemes that have been introduced by the research community till last 5 years. A closer look into the existing scheme shows that there are two forms of multiple access schemes viz. i) orthogonal and ii) non-orthogonal multiple access scheme. The orthogonal access scheme comprises of FDMA, Time Division Multiple Access (TDMA), and combination of both FDMA and TDMA, while the non-orthogonal multiple access schemes could be RSMA, SCMA, and MUSA.

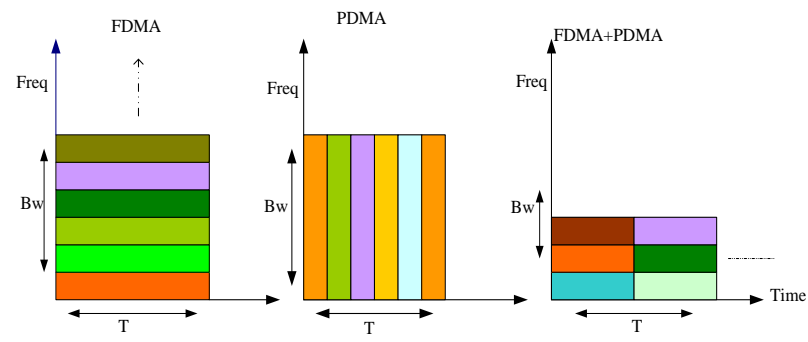

Fig. 15. Classification of Orthogonal Scheme of Multiple Access

Figure.15 highlights the prime distinction among the existing orthogonal schemes of multiple accesses. This section will focus on non-orthogonal schemes as follows:

1) Resource Spread Multiple Access (RSMA)

This scheme make use of the channel coding with minimal rate in order to perform dispersion of the signal over frequency or time domain for accomplishing spectral efficiency. This scheme also ensures proper recovery of the signal even if there is mutual interference. RSMA allows using potential codes for achieve better results. The single carrier RSMA scheme delivers better power efficiency and is independent from any synchronization demands. The multi-carrier RSMA ensures minimal latency when used with devices with low resource capabilities.

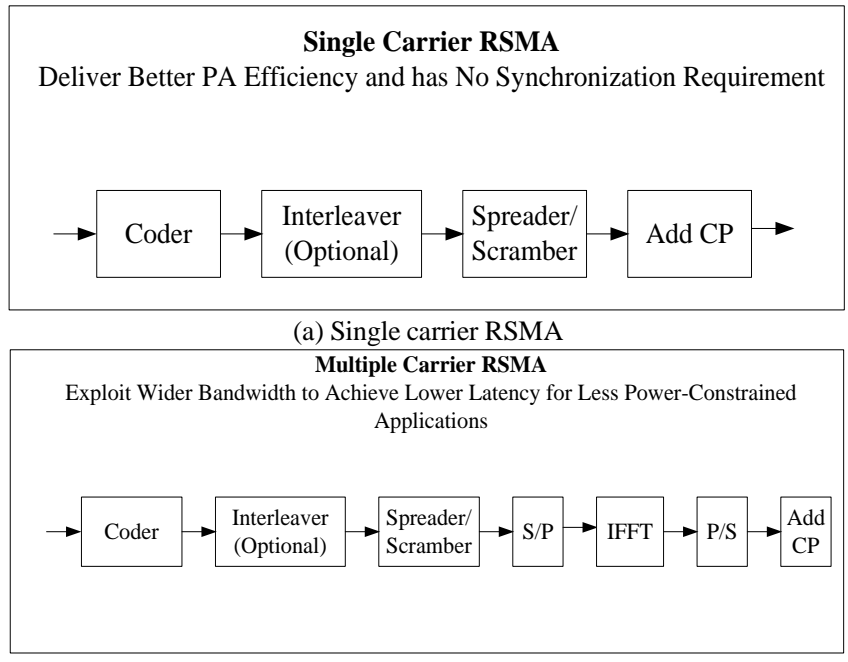

(b) Multiple carrier RSMA

Fig. 16. Working Principle of RSMA

2) Sparse code multiple access (SCMA)

This scheme uses the back end principle of Low Density Signature (LDS) based CDMA for performing i) minimal density spreading and ii) partial deployment of existing time or frequency-based resources. Different from LDS-CDMA, SCMA adopts the working principle of multi-dimensional constellations where a discrete codebook is provided to every users with extensible length of constellation. 


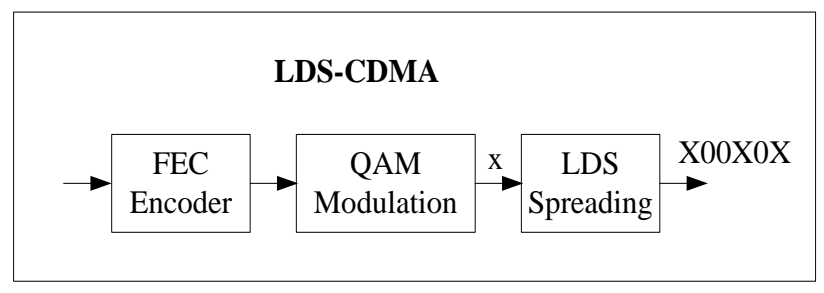

(a) LDS-CDMA

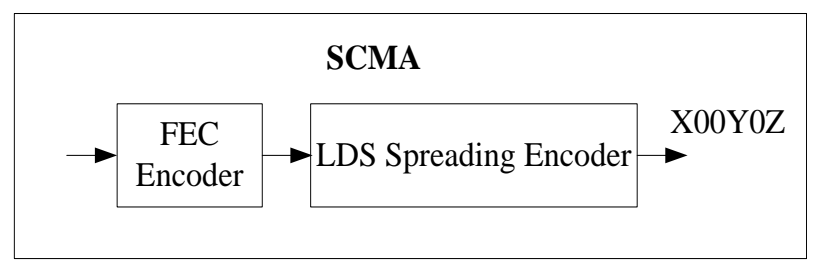

(b) SCMA

Fig. 17. Working Principle of SCMA

\section{STUDY FINDINGS}

Although, OFDM has some problems, but some simple add-ons in the form of waveform or access option can add more benefits to OFDM to support i) maximized spectral efficiency, ii) asynchronous multiplexing, iii) minimal out-ofband emission, iv) minimal complexity, and v) minimal consumption of power.

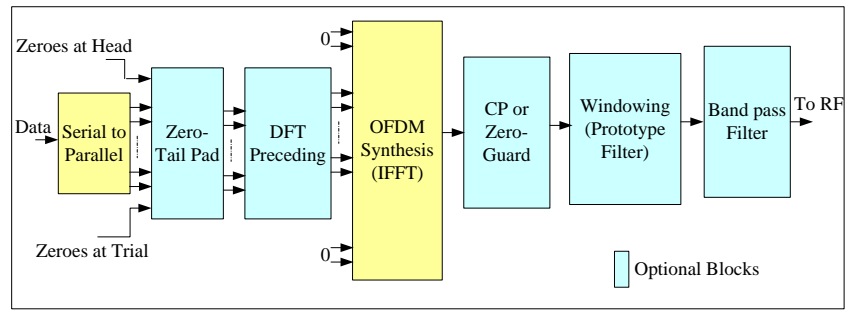

Fig. 18. Multiple Implementation options for OFDM

TABLE II. DIFFERENT OPTIMIZATION OPTIONS FOR OFDM

\begin{tabular}{|l|l|l|l|l|l|l|}
\hline Waveform & A & B & IFFT & C & D & E \\
\hline $\begin{array}{l}\text { CP- } \\
\text { OFDM+WOLA }\end{array}$ & & & $\sqrt{ }$ & CP & $\sqrt{ }$ & \\
\hline $\begin{array}{l}\text { SC- } \\
\text { FDM+WOLA }\end{array}$ & & $\sqrt{ }$ & $\sqrt{ }$ & CP & $\sqrt{ }$ & \\
\hline UFMC & & & $\sqrt{ }$ & ZG & & $\sqrt{ }$ \\
\hline FBMC & & & $\sqrt{ }$ & & $\sqrt{ }$ & \\
\hline $\begin{array}{l}\text { Zero-tail SC- } \\
\text { FDM }\end{array}$ & $\sqrt{ }$ & $\sqrt{ }$ & $\sqrt{ }$ & & & \\
\hline
\end{tabular}

Figure.13 shows multiple optimization schemes carried out towards OFDM e.g. a data is initially subjected to serial-toparallel operation followed by Zero-tail padding (A). It is then subjected to DFT precoding operation (B) followed by IFFT operation. An optional operation of adding cyclic prefix (CP) or Zero Guard (ZG) can be applied (C) followed by windowing (D) and bandpass filter (E) which is then forward in RF. Table 2 summarizes the pictorial representation of Figure.13. The significant research gaps explored are as follows:

- Lower emphasis on spectral efficiency: It was seen that there are certain techniques that has used constant envelop, SC-QAM, SC-FDE etc. These techniques don't guarantee competitive spectral efficiency as they have quite restricted flexibility towards assignment of spectral. Some of them (SC-QAM) doesn't even support MIMO schemes, which shows their incompatibility to be adopted in design mechanism of $5 \mathrm{G}$ technology.

- Complicated uses of Cyclic Prefix: The complimentary waveforms of OFDM are sometime found to include cyclic prefix as well as exclude it on special ground. Although, all the authors gave their own justification, but still it is complicated to understand about the advantage or limitations of using cyclic prefix in new variants of waveforms in $5 \mathrm{G}$. For an example, absence of cyclic prefix makes zero tail SC-FDE to support higher bandwidth allocation but at same time it suffers from dependency towards multiplexing with techniques less than CP-OFDM.

- Partial solution to PAPR and Interference problems: There are hundreds and thousands of literatures where it was claimed that PAPR is solved, but the fact is otherwise. Till date, there is no single standard publication to solve PAPR for waveforms supporting 5G technology. Similarly, effective techniques e.g. GFDM is found to less capable to handle interference owing to its complicated receiver design.

Finally, we conclude our findings by highlighting the effectiveness of various techniques to be used in $5 \mathrm{G}$ in Table 3 .

TABLE III. COMPARISON OF DIFFERENT WAVEFORMS

\begin{tabular}{|l|l|l|l|l|l|l|l|}
\hline $\begin{array}{l}\text { Wave- } \\
\text { forms }\end{array}$ & $\begin{array}{l}\text { SC- } \\
\text { QAM }\end{array}$ & $\begin{array}{l}\text { SC- } \\
\text { FDM/SC- } \\
\text { FDE }\end{array}$ & $\begin{array}{l}\text { Zero- } \\
\text { tail } \\
\text { SC- } \\
\text { FDM }\end{array}$ & $\begin{array}{l}\text { CP- } \\
\text { OFDM } \\
\text { with } \\
\text { WOLA }\end{array}$ & UFMC & FBMC & GFDM \\
\hline W1 & & & & $\sqrt{ }$ & $\sqrt{ }$ & & \\
\hline W2 & $\sqrt{ }$ & $\sqrt{ }$ & $\sqrt{ }$ & $\sqrt{ }$ & $\sqrt{ }$ & $\sqrt{ }$ & $\sqrt{ }$ \\
\hline W3 & $\sqrt{ }$ & $\sqrt{ }$ & & & & & \\
\hline W4 & $\sqrt{ }$ & $\sqrt{ }$ & $\sqrt{ }$ & & & & \\
\hline W5 & $\sqrt{ }$ & $\sqrt{ }$ & $\sqrt{ }$ & $\sqrt{ }$ & & & \\
\hline
\end{tabular}

In the above Table 3, W1 represents supportability of MIMO with maximized spectral efficiency, W2 represents minimized emission due to in-band and out-band signals, W3 represents supportability of asynchronous multiple access, W4 represents lesser extent of consumption of power, and reduced design complexity. It was explored that when OFDM is integrated with different waveforms (e.g. WOLA), the spectral efficiency is found quite maximized with lower design complexity. Hence, there should be enough investigation to enhance the legacy OFDM to include new waveforms that can resist the unsolved problems to be encountered in upcoming $5 \mathrm{G}$ technology.

\section{CONCLUSION}

This paper has presented an elaborated discussion of OFDM and its associated waveforms in order to find their application in next generation of mobile communication standard e.g. 5G. The paper has also reviewed various trends of 5G waveforms e.g. eMBB, OFDM, SC-QAM, SC-FDE, SCFDM, WOLA, zero tail SC-FDM, CP-OFDM, Universal Filtered Multi-Carrier (UFMC), Filter bank Multicarrier (FBMC), and GFDM. The paper has also studied about various multiple access techniques e.g. RSMA and SCMA. The 
content of the present manuscript doesn't want to highlight a negative picture of OFDM but rather it support OFDM and states that there is a larger scope of research in OFDM. Various evolutions of waveforms have been studied, which the research community claims to be supportive of $5 \mathrm{G}$, but unfortunately all of the techniques for generating waveforms are found to possess significant pitfall. This outcome clearly states that existing waveforms doesn't fully support $5 \mathrm{G}$ technology. This finding motivates to continue further research towards OFDM in order to solve the problems existing in present day waveforms and access techniques.

\section{FUTURE WORK}

The future work will be on same direction and will evolve up with a solution to address some of the unsolved problems e.g. power consumption, leakage, interference etc.

\section{REFERENCES}

[1] W.Xiang, K.Zheng, Xuemin (Sherman) Shen, "5G Mobile Communications", Springer Technology \& Engineering, pp. 691, 2016

[2] Y.G. Li, Gordon L. Stuber, "Orthogonal Frequency Division Multiplexing for Wireless Communications", Springer Technology \& Engineering, pp. 308, 2006

[3] W. Abdallah and N. Boudriga, "Enabling 5G wireless access using Li-Fi technology: An OFDM based approach," 2016 18th International Conference on Transparent Optical Networks (ICTON), Trento, 2016, pp. 1-6.

[4] S. Venkatesan and R. A. Valenzuela, "OFDM for 5G: Cyclic prefix versus zero postfix, and filtering versus windowing," 2016 IEEE International Conference on Communications (ICC), Kuala Lumpur, 2016, pp. 1-5.

[5] J. Wang et al., "Nonlinear Inter-Band Subcarrier Intermodulations of Multi-RAT OFDM Wireless Services in 5G Heterogeneous Mobile Fronthaul Networks," in Journal of Lightwave Technology, vol. 34, no. 17, pp. 4089-4103, Sept.1, 12016.

[6] Q. Wang et al., "Enhancing OFDM by Pulse Shaping for Self-Contained TDD Transmission in 5G," 2016 IEEE 83rd Vehicular Technology Conference (VTC Spring), Nanjing, 2016, pp. 1-5.

[7] H. Bogucka, P. Kryszkiewicz and A. Kliks, "Dynamic spectrum aggregation for future 5G communications," in IEEE Communications Magazine, vol. 53, no. 5, pp. 35-43, May 2015.

[8] A. Loulou and M. Renfors, "Enhanced OFDM for fragmented spectrum use in 5G systems", Transactions on Emerging Telecommunications Technologies, Vol. 26, No. 1, pp. 31-45, 2015

[9] C. Y. Lin, Y. C. Chi, C. T. Tsai, H. Y. Wang and G. R. Lin, "39-GHz Millimeter-Wave Carrier Generation in Dual-Mode Colorless Laser Diode for OFDM-MMWoF Transmission," in IEEE Journal of Selected Topics in Quantum Electronics, vol. 21, no. 6, pp. 609-618, Nov.-Dec. 2015.

[10] Z. Li, Y. Chen and X. Zeng, "OFDM synchronization implementation based on Chisel platform for 5G research," 2015 IEEE 11th International Conference on ASIC (ASICON), Chengdu, 2015, pp. 1-4.

[11] H. Lin, "Flexible Configured OFDM for 5G Air Interface," in IEEE Access, vol. 3, no. , pp. 1861-1870, 2015.

[12] P. S. Kildal, X. Chen, M. Gustafsson and Z. Shen, "MIMO Characterization on System Level of 5G Microbase Stations Subject to Randomness in LOS," in IEEE Access, vol. 2, no. , pp. 1062-1075, 2014.

[13] P. Banelli, S. Buzzi, G. Colavolpe, A. Modenini, F. Rusek and A. Ugolini, "Modulation Formats and Waveforms for 5G Networks: Who Will Be the Heir of OFDM?: An overview of alternative modulation schemes for improved spectral efficiency," in IEEE Signal Processing Magazine, vol. 31, no. 6, pp. 80-93, Nov. 2014.

[14] P. Deng and M. Kavehrad, "Real-time software-defined single-carrier QAM mimo visible light communication system," 2016 Integrated Communications Navigation and Surveillance (ICNS), Herndon, VA, 2016, pp. 5A3-1-5A3-11.
[15] T.H. Duyen and T. Pham, "Performance Analysis of MIMO/FSO Systems Using SC-QAM Signaling over Atmospheric Turbulence Channels", IEICE Transactions on Fundamentals of Electronics, Communications and Computer Sciences, Vol. 97, No. 1, pp. 49-56, 2014

[16] J. Zhang, J. Yu, F. Li and N. Chi, "WDM Transmission of SingleCarrier 400G Based on Orthogonal OTDM 80-GBd PDM-8QAM," in IEEE Photonics Journal, vol. 7, no. 4, pp. 1-6, Aug. 2015.

[17] F. C. Ribeiro, R. Dinis, F. Cercas and A. Silva, "Clustered Multiuser Detection for the Uplink of 5G Systems," 2016 IEEE 83rd Vehicular Technology Conference (VTC Spring), Nanjing, 2016, pp. 1-5.

[18] R. Dinis and P. Montezuma, "Iterative receiver based on the EGC for massive MIMO schemes using SC-FDE modulations," in Electronics Letters, vol. 52, no. 11, pp. 972-974, 5262016.

[19] B. Zheng; F. Chen; M. Wen; F. Ji; H. Yu, "Novel Pilot Position Selection and Signal Reconstruction Methods for Frequency Domain Pilot Multiplexing Techniques of SC-FDE," in IEEE Transactions on Vehicular Technology, vol.PP, no.99, pp.1-1

[20] M. Wu, D. Wubben, A. Dekorsy, P. Baracca, V. Braun and H. Halbauer, "On OFDM and SC-FDE Transmissions in Millimeter Wave Channels with Beamforming," 2016 IEEE 83rd Vehicular Technology Conference (VTC Spring), Nanjing, 2016, pp. 1-5

[21] X. Cheng, Z. Luo and S. Li, "Joint Estimation for I/Q Imbalance and Multipath Channel in Millimeter-Wave SC-FDE Systems," in IEEE Transactions on Vehicular Technology, vol. 65, no. 9, pp. 6901-6912, Sept. 2016.

[22] A. Boonkajay and F. Adachi, "Low-PAPR joint transmit/received SCFDE transmission using time-domain selected mapping," The 20th AsiaPacific Conference on Communication (APCC2014), Pattaya, 2014, pp. 248-253.

[23] M. Zhao, F. Yang, L. Ding, Y. Guan and L. Qian, "Research on Tone Reservation in SC-FDM system," 2016 22nd Asia-Pacific Conference on Communications (APCC), Yogyakarta, 2016, pp. 394-399.

[24] X. Luo, "Low-PAPR Multiplexing of Data and Pilots," 2016 IEEE 83rd Vehicular Technology Conference (VTC Spring), Nanjing, 2016, pp. 1-5.

[25] J. Zhou et al., "Coherent Optical Interleaved SC-FDM Uplink Scheme for Long-Reach Passive Optical Network," in IEEE Photonics Journal, vol. 8, no. 2, pp. 1-8, April 2016.

[26] T. Kobayashi, A. Sano, A. Matsuura, Y. Miyamoto and K. Ishihara, "Nonlinear Tolerant Spectrally-Efficient Transmission Using PDM 64QAM Single Carrier FDM With Digital Pilot-Tone," in Journal of Lightwave Technology, vol. 30, no. 24, pp. 3805-3815, Dec.15, 2012.

[27] A. V. Dorokhov, V. V. Geppener, V. V. Gulvanskiy, D. I. Kaplun and D. M. Klionskiy, "Multichannel filter bank implementation and prototype-filter design based on magnitude response symmetrization," Soft Computing and Measurements (SCM), 2015 XVIII International Conference on, St. Petersburg, 2015, pp. 96-99.

[28] F. Frey, R. Elschner, C. Kottke, C. Schubert and J. K. Fischer, "Efficient real-time implementation of a channelizer filter with a weighted overlapadd approach," 2014 The European Conference on Optical Communication (ECOC), Cannes, 2014, pp. 1-3.

[29] D. M. Klionskiy, D. I. Kaplun, A. S. Voznesenskiy and V. V. Gulvanskiy, "Multichannel WOLA algorithm in hydroacoustic monitoring and radio monitioring tasks and its computer simulation in MATLAB," Emission Electronics (ICEE), 2014 2nd International Conference on, St. Petersburg, 2014, pp. 1-6.

[30] S. C. Lai, H. C. Lai, F. C. Hong, H. R. Lin and S. F. Lei, "A Novel Coherence-Function-Based Noise Suppression Algorithm by Applying Sound-Source Localization and Awareness-Computation Strategy for Dual Microphones," Intelligent Information Hiding and Multimedia Signal Processing (IIH-MSP), 2014 Tenth International Conference on, Kitakyushu, 2014, pp. 313-316.

[31] L. Tao, W. Zhigang, W. Tao, G. Lianpin and L. Guangkun, "Flexible approach to WOLA-Based wideband IF signal analysis," Electronic Measurement \& Instruments (ICEMI), 2013 IEEE 11th International Conference on, Harbin, 2013, pp. 192-196.

[32] G. Berardinelli, F. Frederiksen, K. Pedersen, P. Mogensen and K. Pajukoski, "Reference sequence design for zero-tail DFT-spread- 
OFDM," 2016 IEEE Wireless Communications and Networking Conference, Doha, 2016, pp. 1-6.

[33] G. Berardinelli, K. I. Pedersen, F. Frederiksen, P. Mogensen and K. Pajukoski, "A novel channel estimator for Zero-Tail DFT-spreadOFDM," 2016 International Symposium on Wireless Communication Systems (ISWCS), Poznan, Poland, 2016, pp. 373-377.

[34] G. Berardinelli, F. M. L. Tavares, T. B. Sorensen, P. Mogensen and K. Pajukoski, "On the Potential of Zero-Tail DFT-Spread-OFDM in 5G Networks," 2014 IEEE 80th Vehicular Technology Conference (VTC2014-Fall), Vancouver, BC, 2014, pp. 1-6.

[35] P.F. Pazos, "Waveform evaluation for 5G Networks", PhD diss., Aalborg University, 2015

[36] R. Ahmed, T. Wild and F. Schaich, "Coexistence of UF-OFDM and CPOFDM," 2016 IEEE 83rd Vehicular Technology Conference (VTC Spring), Nanjing, 2016, pp. 1-5.

[37] Q. Bodinier, F. Bader and J. Palicot, "Modeling interference between OFDM/OQAM and CP-OFDM: Limitations of the PSD-based model," 2016 23rd International Conference on Telecommunications (ICT), Thessaloniki, 2016, pp. 1-7.

[38] M. Renfors, J. Yli-Kaakinen, T. Levanen, M. Valkama, T. Ihalainen and J. Vihriala, "Efficient Fast-Convolution Implementation of Filtered CPOFDM Waveform Processing for 5G," 2015 IEEE Globecom Workshops (GC Wkshps), San Diego, CA, 2015, pp. 1-7.

[39] M. Bellanger, D. Mattera and M. Tanda, "Lapped-OFDM as an Alternative to CP-OFDM For 5G Asynchronous Access and Cognitive Radio," 2015 IEEE 81st Vehicular Technology Conference (VTC Spring), Glasgow, 2015, pp. 1-5.

[40] B. Chen, Z. Zhang, H. Yue and Y. Zhang, "Reaserch on synchronization issues of CP-OFDM receiver," Electronics Information and Emergency Communication (ICEIEC), 2015 5th International Conference on, Beijing, 2015, pp. 126-130.

[41] D. Katselis, "Some Preamble Design Aspects in CP-OFDM Systems," in IEEE Communications Letters, vol. 16, no. 3, pp. 356-359, March 2012.

[42] T. van Waterschoot, V. Le Nir, J. Duplicy and M. Moonen, "Analytical Expressions for the Power Spectral Density of CP-OFDM and ZPOFDM Signals," in IEEE Signal Processing Letters, vol. 17, no. 4, pp. 371-374, April 2010.

[43] H. Kim, J. Bang, S. Choi and D. Hong, "Resource block management for uplink UFMC systems," 2016 IEEE Wireless Communications and Networking Conference, Doha, 2016, pp. 1-4.

[44] C. Vitiello et al., "Two-step resource allocation for BIC-UFMC wireless communications," 2016 International Symposium on Wireless Communication Systems (ISWCS), Poznan, Poland, 2016, pp. 378-382.

[45] L. Zhang, P. Xiao and A. Quddus, "Cyclic Prefix-Based Universal Filtered Multicarrier System and Performance Analysis," in IEEE Signal Processing Letters, vol. 23, no. 9, pp. 1197-1201, Sept. 2016.

[46] Suiyan Geng, Xin Xiong, Linlin Cheng, Xiongwen Zhao and Biao Huang, "UFMC system performance analysis for discrete narrow-band private networks," 2015 IEEE 6th International Symposium on
Microwave, Antenna, Propagation, and EMC Technologies (MAPE), Shanghai, 2015, pp. 303-307.

[47] M. Mukherjee, L. Shu, V. Kumar, P. Kumar and R. Matam, "Reduced out-of-band radiation-based filter optimization for UFMC systems in 5G," 2015 International Wireless Communications and Mobile Computing Conference (IWCMC), Dubrovnik, 2015, pp. 1150-1155.

[48] F. Schaich and T. Wild, "Waveform contenders for 5G - OFDM vs. FBMC vs. UFMC," Communications, Control and Signal Processing (ISCCSP), 2014 6th International Symposium on, Athens, 2014, pp. 457460.

[49] H. Hosseini, A. Anpalagan, K. Raahemifar, S. Erkucuk and S. Habib, "Joint wavelet-based spectrum sensing and FBMC modulation for cognitive mmWave small cell networks," in IET Communications, vol. 10 , no. 14 ,

[50] H. Jamal; D. W. Matolak, "FBMC and LDACS Performance for Future Air to Ground Communication Systems," in IEEE Transactions on Vehicular Technology, vol.PP, no.99, pp.1-1

[51] X. Mestre and D. Gregoratti, "Corrections to "Parallelized Structures for MIMO FBMC Under Strong Channel Frequency Selectivity" [Mar 16 1200-1215]," in IEEE Transactions on Signal Processing, vol. 64, no. 17, pp. 4644-4644, Sept.1, 12016.

[52] D. Na and K. Choi, "Intrinsic ICI-Free Alamouti Coded FBMC," in IEEE Communications Letters, vol. 20, no. 10, pp. 1971-1974, Oct. 2016.

[53] F. Rottenberg; X. Mestre; F. Horlin; J. Louveaux, "Single-Tap Precoders and Decoders for Multi-User MIMO FBMC-OQAM under Strong Channel Frequency Selectivity," in IEEE Transactions on Signal Processing, vol.PP, no.99, pp.1-1

[54] D. Sim, K. Kim and C. Lee, "A Layered Detection Algorithm Based on Interference Cancellation for FBMC-QAM," in IEEE Communications Letters, vol. 20, no. 10, pp. 1939-1942, Oct. 2016.

[55] L. Chang, G. Y. Li, J. Li and R. Li, "Blind Parameter Estimation of GFDM Signals Over Frequency-Selective Fading Channels," in IEEE Transactions on Communications, vol. 64, no. 3, pp. 1120-1131, March 2016.

[56] Z. Sharifian, M. J. Omidi, H. Saeedi-Sourck and A. Farhang, "Linear Precoding for PAPR Reduction of GFDMA," in IEEE Wireless Communications Letters, vol. 5, no. 5, pp. 520-523, Oct. 2016.

[57] D. W. Lin and P. S. Wang, "On the Configuration-Dependent Singularity of GFDM Pulse-Shaping Filter Banks," in IEEE Communications Letters, vol. 20, no. 10, pp. 1975-1978, Oct. 2016.

[58] P. S. Wang and D. W. Lin, "Maximum-Likelihood Blind Synchronization for GFDM Systems," in IEEE Signal Processing Letters, vol. 23, no. 6, pp. 790-794, June 2016.

[59] P. Wei, X. G. Xia, Y. Xiao and S. Li, "Fast DGT-Based Receivers for GFDM in Broadband Channels," in IEEE Transactions on Communications, vol. 64, no. 10, pp. 4331-4345, Oct. 2016.

[60] D. Zhang, L. L. Mendes, M. Matthé, I. S. Gaspar, N. Michailow and G. P. Fettweis, "Expectation Propagation for Near-Optimum Detection of MIMO-GFDM Signals," in IEEE Transactions on Wireless Communications, vol. 15, no. 2, pp. 1045-1062, Feb. 2016. 